\title{
¿Cabe una educación sexual que sea expresión de una inteligencia cultivada?
}

\section{Is there a sexual education that is an expression of a cultivated intelligence?}

Dr. David REYERO. Profesor Titular. Universidad Complutense de Madrid (reyero@ucm.es).

\section{Resumen:}

El presente texto pretende mostrar cómo la educación sexual en la actualidad responde al dominio que las filosofías de la sospecha han conseguido sobre la vida intelectual. Este dominio dificulta un juicio normativo sobre la sexualidad humana. Cualquier intento de normatividad es sospechoso de esconder algún tipo de dominación de unos sobre otros. La ausencia de normatividad imposibilita una educación de la sexualidad significativa y solo permite una educación superficial, instrumental y limitada al manejo de las consecuencias no deseadas: embarazos adolescentes, enfermedades de transmisión sexual, etc. Mostramos la existencia de otra forma de entender la vida intelectual ligada a la vida moral, abierta a la verdad y no solo dedicada a la denuncia. Pensar bien implica vivir en el seno de una tradición y en el camino de la vida buena. Una idea de vida buena supone la comprensión teleológica de la condición humana y el necesario cul- tivo de unas virtudes para mantenerse en ella. En esa dimensión, la sexualidad tiene un sentido no meramente biológico sino relacional, generativo y comunicativo, y está sometida a unas reglas fruto de nuestro carácter personal y de la relación que la sexualidad tiene con la intimidad.

Descriptores: educación sexual, pensamiento, educación de la afectividad, educación moral.

\section{Abstract:}

This article aims to show how sex education today responds to the dominance that philosophies of suspicion have achieved over intellectual life, a dominance that hinders a normative judgment of human sexuality. Any attempt at regulation is suspected of concealing some kind of domination by some over others. The lack of regulations makes meaningful education about sexuality

Fecha de recepción de la versión definitiva de este artículo: 28-09-2020.

Cómo citar este artículo: Reyero, D. (2021). ¿Cabe una educación sexual que sea expresión de una inteligencia cultivada? | Is there a sexual education that is an expression of a cultivated intelligence? Revista Española de Pedagogía, 79 (278), 115-129. https://doi.org/10.22550/REP79-1-2021-05 
impossible and only allows for education that is superficial, instrumental, and limits itself to managing unwanted consequences such as teenage pregnancies and sexually transmitted infections. This work shows that there is another way of understanding intellectual life that is linked to moral life, open to the truth, and not just dedicated to denunciation. Thinking well involves living within a tradition and on the path of a good life. A concept of a good life supposes a teleological understanding of the human condition and the necessary cultivation of virtues in order to remain within it. In this dimension, sexuality has a meaning that is not merely biological but also relational, generative, and communicative, and it is subject to rules that derive from our personal character and sexuality's relationship with intimacy.

Keywords: sex education, thinking, affective education, moral education.

\section{La vida intelectual}

En 1975 Robert Spaemann escribió un interesante artículo titulado «iEs la emancipación un objetivo para la educación?» El artículo era provocador, pues el concepto emancipación estaba de moda entonces y sigue estándolo cuando hablamos de los fines de la educación. La respuesta sorprendente y rápida es un rotundo no, aunque habremos de matizarla ahora porque, ciertamente, Spaemann no está proponiendo una pedagogía para el dominio, ni tampoco un ideal de ser humano subyugado. El enemigo al que Spaemann se enfrenta es una concreta manera de entender el pensamiento cuyo fundamento último es la sospecha y la desconfianza. Según entendamos la función del pensamiento humano, así entenderemos su relación con la educación y las distintas dimensiones de la persona. Para los ideólogos de la emancipación, todo debe partir de un lento proceso de autoconciencia de aquellos aspectos de nosotros mismos que responden a intereses sobre los que no somos dueños, y que son el fruto de una tradición. Esa tradición nos ofrece sentido, un modo de vida y un lenguaje con el que explicarnos a nosotros mismos y también la realidad que nos rodea. Pero dicho lenguaje también oculta o justifica relaciones asimétricas y distribuciones desiguales de poder. Parecería, según estas filosofías de la sospecha, que el ser humano es, incluso cuando no lo sabe, esclavo, pues está inserto en relaciones que él no ha elegido y sobre las que no siempre tiene posibilidades de emancipación. Todo interés es, por lo tanto, sospechoso y es la labor del pensamiento desvelar lo que está oculto a la vista. Esa manera de entender el pensamiento ha tenido también su traducción en el ámbito de la pedagogía a través de lo que se conoce como pedagogía crítica. Para la pedagogía crítica la función de este tipo de conocimiento, que conocemos como pedagogía, no es el diseño de las estrategias para la transmisión de un conocimiento establecido, sino que «la pedagogía crítica ilumina las relaciones entre conocimiento, autoridad y poder. Por ejemplo, plantea preguntas 
acerca de quién tiene control sobre las condiciones en las que se produce el conocimiento» (Giroux, 2013, p. 15).

La pedagogía crítica supone la sospecha de toda relación asimétrica que se basa en una autoridad o una tradición. El objetivo es lo que se conoce como democracia radical. Esta democracia es el fruto de una tarea pedagógica previa que deconstruya toda ideología que nos configura, pues «las ideologías no son sólo una constelación de ideas, estereotipos, y modos de sentido común; también representan formas específicas de conocimiento y creencias arraigadas en fuertes cargas emocionales. Es necesario comprender, analizar, y de-construir este tipo de vinculaciones, a menudo no ya como una forma de conocimiento incomprensible, sino como un rechazo activo a saber y el rechazo a reconocer lo implicado que uno puede estar en este tipo de vinculaciones» (Giroux, 2013, p. 22). Por lo tanto, toda ideología engendra en su interior algún tipo de opresión contra quien no quiere 0 puede someterse a sus categorías y a las normas que estas traen siempre consigo.

El papel del pensamiento es, por lo tanto, desvelar, o mejor, denunciar, porque tras el sentido hay siempre interés oculto. Esta forma de orientar el cultivo de la inteligencia resulta claramente insuficiente, aunque pueda parecer atractiva, pues nacería del reconocimiento de que lo valioso del pensamiento está en su capacidad de desvelar/denunciar el error, el abuso o la mentira, más que en desvelar/descubrir lo valioso, lo «interesante», lo bueno o lo verdadero.
La razón de esta forma de entender el pensamiento y, por lo tanto, el valor de la vida intelectual descansa en dos errores. El primero lo situaremos en una cierta incomprensión del carácter limitado de la vida y el pensamiento humano. Ante él se nos aparece el atractivo de la novedad como un escape frente al aburrimiento 0 cansancio que sobreviene, tantas veces, a lo habitual. En efecto, pensar no es simplemente la concatenación lógica de ideas y argumentos, sino que es también introducirse en una tradición con unas prácticas concretas y unas determinadas exigencias que afectan, también, a la forma de vida y los hábitos que nos mantienen en ella. Pensar tiene, por lo tanto, relación con esa segunda naturaleza que llamamos vida moral y no solo con la lógica. Esa segunda naturaleza se construye laboriosamente y se sustenta siempre en un lenguaje de sentido cuya veracidad no puede descubrirse si no se vive en él.

Este es el sentido de la crítica que, por ejemplo, MacIntyre (2001) realiza al concepto de educación crítica que tiene Richard Rorty. Para Rorty el pensamiento formado, el pensamiento crítico es el que es capaz de ironizar una vez ha sido socializado en un modo de ver la realidad. Ciertamente, para ser justos hay que reconocer que, según Rorty, no existe posibilidad de pensar críticamente si antes no nos hemos hecho con un determinado tipo de lenguaje y comprensión del mundo, lo que él liga a la socialización (Rorty, 1990). Pero para Rorty, lo característico del ser humano es la posibilidad de poner en cuestión, con todas las consecuencias, ese pensamiento último, ironizar creativamente con él. 
Frente a esta manera dominante de entender la vida intelectual, autores como MacIntyre ofrecen alternativas. Esencialmente, el ser humano es un ser profundamente dependiente cuyo telos es conseguir ser independiente, o tener un pensamiento autónomo, o pensamiento propio o crítico. Lo peculiar para MacIntyre de ese viaje a la independencia es que no puede hacerse sino desde la dependencia. Podría parecer que estamos hablando de lo mismo que cuando en Rorty hablábamos de la necesidad de la socialización, pero, sin embargo, no es lo mismo. Para MacIntyre la dependencia es una condición de posibilidad para el pensamiento autónomo o lo que, por usar su terminología, él llama «razonamiento práctico independiente». Llegamos a ser autónomos gracias a los demás, no a pesar de los demás. Esto tiene una implicación importante: la desconfianza frente a lo recibido no es el objetivo de la vida humana y ni siquiera es algo que sea esencialmente deseable. Dice MacIntyre (2001) que hay que tener muy buenas razones para oponernos a los que nos quieren y nos han enseñado a ser quienes somos. Y aunque quizás a veces hay que hacerlo, y parte importante de la educación consiste en ese difícil aprendizaje, siempre hay que tener muy buenas razones para hacerlo.

MacIntyre quiere destacar la importancia de la formación intelectual en el seno de una tradición, la idea de que el aprendizaje de lo que somos es siempre gracias a los demás, y que la tradición es algo cualitativamente distinto a la socialización. MacIntyre se decide a explorar lo que él llama virtudes de la dependencia, el tipo de actitudes que permiten una mejor vida social. Entre las virtudes necesarias destaca la veracidad. Es importante recordar aquí que para Rorty todo discurso sobre la veracidad resultaba inconveniente, siendo para MacIntyre fundamental, no solo para el pensamiento, entendido como pura cognición, sino también para la formación del juicio moral.

Para MacIntyre hay tres actitudes que dañan la necesaria virtud de la veracidad: «Una primera agresión contra la veracidad consiste en impedir que los demás aprendan injustamente lo que necesitan aprender, y una segunda, en ocultar la naturaleza de las relaciones que uno tiene con los demás» (MacIntyre, 2001, p. 177). La tercera agresión de la que habla MacIntyre es precisamente la ironía de Rorty, que identifica con la actitud sofista incapaz de comprometerse con un vocabulario final. ¿Por qué? Primero, porque un vocabulario final no es una suerte de socialización superficial de costumbres que nos permite aparecer ante los otros y ser reconocidos, sino también un vocabulario que nos compromete, nos explica, nos posibilita entender el mundo humano, y permite su funcionamiento. ¿Cómo funcionaría una sociedad si no podemos fiarnos realmente los unos de los otros, o si no creemos que entendemos lo mismo, en una gran medida al menos, acerca de lo justo, lo bueno y lo verdadero? ¿Cómo podríamos siquiera comprar en el supermercado sin fiarnos de la veracidad de lo que nos dicen? ¿Deberíamos desconfiar de que nos están vendiendo un litro de leche cuando nos dicen que nos están vendiendo un litro de leche? o ipor qué acudir a clase y fiarnos de un profesor si no pensáramos que lo que nos dice es esencialmente cierto? El desapego irónico 
es para MacIntyre un cinismo que haría de la vida social una vida invivible. Si el desapego irónico es genuino y no una mera pose, si pone verdaderamente en cuestión las bases en las que nos han socializado, estaríamos al albur del más fuerte, del más poderoso o del que tiene más recursos para imponer su agenda.

Para MacIntyre no siempre la crítica es necesaria 0 valiosa para avanzar en el conocimiento. MacIntyre critica la idea de situar en el campo de la desconfianza y la duda el fundamento de la vida intelectual. El argumento esencial de MacIntyre tiene que ver con el progreso en la virtud. Utilizaremos un ejemplo: si nos preguntásemos acerca del por qué no traicionar a un amigo si nos conviene, significaría que estábamos aún en un nivel de comprensión muy inicial en torno a lo que significa la amistad. El que progresa en el cultivo de esa virtud llegará un momento en el que no podrá hacerse esa pregunta, y esa imposibilidad es un logro que le confiere un pensamiento mejor y más perfecto, y le sitúa ante la posibilidad de tener un juicio más fino con respecto a la práctica de la amistad.

En resumen, la vida intelectual cultivada no es aquella que nace de la desconfianza o la puesta en cuestión, porque cuestionar nuestros intereses, su historia y genealogía no es suficiente, ni siquiera es útil. Necesitamos tener intereses y esos intereses se consiguen de otra manera. Una vida plenamente humana es una vida con intereses (Spaemann, 2003). La verdadera vida intelectual es la que nace de la profundización y mejora en la búsqueda de bienes o prácticas verdaderamente valiosas, y ese pensamiento requiere de práctica, de un estar sumergido de lleno en un determinado tipo de vida sea esta científica, filosófica, humanista o religiosa. Pensar bien implica vivir con un determinado estilo y no solo razonar de una determinada manera. Desde este punto de vista podemos entender que fenómenos, como la comunión, tener un mismo sentir y una misma mentalidad son la consecuencia de una determinada vida intelectual marcada por la experiencia del conocimiento compartido fruto de una misma tradición. Un conocimiento,

per connaturalitatem así como se conoce lo querido o lo propio; el extraño no comprende nada o lo malentiende, pero el unido en amor o parentesco esencial sabe inmediatamente y con absoluta seguridad lo que se quiere decir en un fragmento de una carta 0 en una llamada oída solo confusamente (Pieper, 2005, p.71).

Esta idea nos lleva al segundo de los errores de este pensamiento de la sospecha. Los modelos nacidos de la sospecha desembocan, paradójicamente, en la exaltación de lo aparentemente creativo $\mathrm{u}$ original $\mathrm{y}$ también de lo identitario y lo sentimental. La postmodernidad identitaria es el hijo inevitable de la modernidad racionalista, que es la madre de las filosofías de la sospecha.

Como acabamos de ver, la modernidad, origen del pensamiento crítico del que estamos hablando, trató de romper amarras con la tradición y con una forma de pensar que nace de una observación teleológica profunda del ser humano. Esa ruptura ha supuesto un duro golpe para la formación del pensamiento que ahora no tiene más criterio que su propia originalidad y

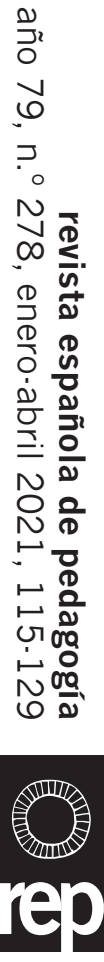


la capacidad de seducción. La validez del pensamiento está ahora en la creatividad que muestra 0 en su capacidad de entretener o deslumbrar, pero no en su poder para desentrañar la naturaleza humana. Es, en este sentido, muy común que, por ejemplo, los adolescentes queden cautivados por series de moda que, bajo un disfraz atractivo, ofrecen cauces al deseo a la vez que oscurecen aspectos importantes de la naturaleza humana.

Si todo lo que queda es sospechar, solo podemos recurrir a la experiencia de nuestro propio deseo que, sin criterio de juicio ni referencia fiable, es lo único que aparece como verdaderamente real. ¿Qué hago yo con mi deseo que se me presenta a mí como cuando me duele algo, reclamando en todo momento mi atención? En ausencia de otros criterios sustantivos solo queda ofrecer a ese deseo toda la primacía, y siempre que no se atente contra otros, parece lo más racional. En consecuencia, la vida intelectual es una vida que acompaña 0 encuentra razones para justificar 0 legitimar ese deseo.

\section{La formación de la sexualidad humana y la tradición}

Hay una fuerte relación entre la vida intelectual que nace de las filosofías de la sospecha y la concepción actual de la sexualidad y su formación. La sexualidad en el mundo actual arriba descrito es objetivo de la educación solo en la medida en que preocupan sus consecuencias más negativas: los embarazos adolescentes, las enfermedades de transmisión sexual, la consideración de las diferentes identidades y orientaciones sexuales o el patriarcado que hace referen- cia a la supuesta desigualdad en la distribución del poder en las relaciones entre hombres y mujeres. En cualquier caso, podremos estudiar la sexualidad siempre que no entremos en el terreno del comportamiento correcto o incorrecto, terminología de la que habremos de sospechar. Pero hablar de la sexualidad en la escuela nunca ha sido sencillo, ni aquí ni en otros sitios. Esto es así porque el debate acerca de lo que la escuela puede o debe decir al respecto está atravesado de concepciones antropológicas de fondo y rivales (Irvine, 2004).

Así, cuando, por ejemplo, se contraponen las dos corrientes más clásicas en los Estados Unidos de educación sexual Abstinence Only y Comprehensive Sex Education late en el fondo una muy diferente concepción de lo valioso y lo moral. Para los primeros, las relaciones sexuales completas fuera del matrimonio resultan inmorales per se, y la información sobre métodos para evitar el embarazo puede resultar contraproducente, pues incita a los adolescentes a la realización de actividades sexuales para las que no están psicológicamente preparados, que resultan moralmente rechazables y que, por su propia naturaleza, pueden dar lugar a todo aquello que también sus rivales quieren evitar, enfermedades de transmisión sexual o embarazos adolescentes, con las consecuentes caídas en la pobreza, marginación, etc.

Por contra, los defensores de lo que los americanos llaman método comprensivo de educación sexual señalan que no es cierto que la información sobre distintos métodos de contracepción incite a una más temprana actividad sexual. Argumentan también 
que hay un derecho a la información sexual y a la formación en las prácticas que permitan un «sexo seguro». Su razonamiento último es que vivimos en una sociedad plural y no todos comparten las mismas concepciones antropológicas de fondo, y lo que debemos solucionar no es tanto un problema moral, que, si acaso lo será para algunos, cuanto uno de salud pública en el que todos estamos de acuerdo (Fine, 1988; Fine y McClelland, 2006; Alagiri, Summers y Morin, 2002; Santelli et al., 2017). Dentro de esta misma corriente hay otra serie de estudios que pretenden ir más allá de la mirada centrada en la dimensión del sexo adolescente como actividad de riesgo, para centrarse en una consideración positiva, dicen ellos, de la sexualidad adolescente, destacando su importancia como fuerza individualizadora, y como espacio para enfrentar los modelos dominantes en las relaciones entre hombres y mujeres (Tolman, 2012; Tolman y McClelland, 2011; Harden, 2014). Pero, por muy positivo que consideren su acercamiento, sus análisis se mantienen en una dimensión sociologista 0, como mucho, psicologista del fenómeno de la sexualidad humana. Jamás entran en la dimensión antropológica o de sentido.

El problema de ambos métodos es que los dos tropiezan con la misma piedra, que es en el fondo intelectual, y que podríamos denominar puritanismo o simplicidad. Entendemos el término puritanismo en el sentido en el que Roger Scruton lo entiende; para este autor, el puritanismo es una forma de pereza que ante un problema complejo pretende sortearlo por la vía fácil (Scruton, 2017, p. 211). Como la educación de la afectividad, en la que está inscrita la educación del deseo sexual, es un asunto complejo que requiere de un concienzudo y sostenido trabajo, pues apliquemos la solución fácil de la pura negación del deseo 0 tratemos los aspectos más desagradables e inconvenientes de su desbocamiento. Los que abogan por la vía de la simple propuesta de la abstinencia son perezosos si se quedan solo aquí, pues pretenden resolver un problema sin ayudar al cultivo de las virtudes necesarias para encauzar la afectividad, ni mostrar la belleza de su consecución. Los resultados efectivos parecen así poco eficaces (Santelli et al., 2017).

Por otra parte, aquellos que pretenden solventar el asunto de la educación sexual simplemente mediante el control técnico de las consecuencias no deseadas, a través de la información sobre preservativos y «prácticas seguras», también manifiestan pereza, pues rechazan voluntariamente entrar en el significado del deseo sexual para el ser humano y rompen, así, cualquier tipo de vinculación con los significados que para nuestra tradición ha tenido la sexualidad. Su propuesta resulta también perezosa en la medida en que dejan al adolescente sin referentes para entender su deseo y cómo afrontarlo.

Ambos se manejan entre los restos de una comprensión en un mundo en el que se ha roto la relación entre sexualidad, estabilidad, amor y reproducción. Un mundo en el que prima la facilidad de acceso a métodos contraceptivos, y una absoluta disponibilidad de sexo en la red.

Para enfrentar el tema de la sexualidad humana y cómo puede ayudar a la forma- 
ción de su dirección el cultivo de la vida intelectual, es necesario comprender que existe una manera de aproximarse a esta dimensión de la persona que no surge de la pedagogía de la sospecha, ni de la constatación de la existencia de un deseo que no hay por qué reprimir, siempre que se haga con el único límite de no provocar daño al otro. Surge, más bien, de la capacidad de aprender de la historia y la experiencia atenta buscando el sentido de nuestra realidad afectivo sexual. ¿Qué podemos aprender de esa observación?

Primero, la sexualidad es una dimensión importante que hay que afrontar lejos de la actitud puramente defensiva y miedosa con la que se ha podido afrontar educativamente en otras épocas, pero sin caer en su banalización. La sexualidad forma parte importante en los procesos de individualización y crecimiento humano y es, en cierto sentido, sagrada para el ser humano, en la medida en la que une dos dimensiones: la intimidad y la generación de nueva vida.

Que la sexualidad tiene relación con la intimidad es evidente solo con observar las reacciones que nos genera la violencia sexual. Esta nos provoca un mayor rechazo que otros tipos de violencia porque entendemos que la violencia sexual es un ataque a la intimidad que marca de manera profunda a las personas que la sufren. Sin esa relación entre sexualidad e intimidad sería difícil explicar por qué nos puede producir más rechazo una simple mirada que una pelea en el patio de instituto por una disputa deportiva. Por otra parte, la relación entre sexualidad y reproducción es evidente, aunque se intente ocultar. Por más que hoy en día hablemos de sexo seguro como de aquel que no puede producir un embarazo, lo que es seguro es que todos venimos de una relación sexual. Es en esta unión de la intimidad con la reproducción donde radica el origen de la continua regulación de la sexualidad que las distintas culturas humanas han ejercido sobre la misma. Esa «sacralidad» de la sexualidad humana se manifiesta en la enorme cantidad de normas y ritos que históricamente han regulado su funcionamiento. No hay función corporal más sometida a institucionalización que la relacionada con la sexualidad. Como dice Spaemann, ese carácter esencial de la sexualidad humana, que se distingue de otras formas de satisfacción de las necesidades, «está sometida a ciertas reglas de humanización y tiene el privilegio que proviene de su institucionalización» (Spaemann, 2017, p. 29). La institucionalización es la manera humana de sellar aquellas prácticas que tienen una relevancia social.

Sin embargo, no podemos obviar que en el último medio siglo se ha producido un intenso y progresivo proceso de privatización del sexo, o desinstitucionalización, que corre paralelo a la también progresiva separación entre sexualidad y reproducción. Dicha separación o ruptura se acrecienta gracias a los medios de control de la natalidad, y a la revolución que estos han supuesto para la vida humana (Choza, 2020, p. 138-141). Para este autor, esta ruptura entre sexo y reproducción libera al sexo, que ya no tiene consecuencias sociales negativas, siempre que se respete la voluntad del otro. En consecuencia, para Choza, la vieja arquitectura moral montada en torno a la relación entre sexo y 
reproducción carece ya de sentido y resulta difícil de transmitir. Este autor entiende que en su globalidad hay una ganancia en este proceso (Choza, 2020, p. 273-276).

Choza anticipa, quizá prematuramente, que la ruptura entre reproducción y sexualidad no va a tener efectos sobre la intimidad. Sin embargo, esa anticipación puede no ser cierta tal y como nos empiezan a mostrar las redes y algunos fenómenos sociales actuales ${ }^{1}$.

En un reciente ensayo publicado por Mary Eberstadt (2020), la autora defiende que los problemas que surgen del auge de las políticas de la identidad, y que han dado lugar a una enorme polarización y explosiones violentas, también dentro de las instituciones académicas, tienen su raíz última en la revolución sexual global y la ruptura que ha supuesto para la familia. El ensayo remite al famoso libro de Alan Bloom The Closing of the American Mind (El cierre de la mente moderna) (2008). En ese libro, Bloom se queja de la deriva relativista hacia la que se ha escorado la educación superior. Una de las causas que Bloom encontraba de este escoramiento al relativismo tenía que ver con el aumento de los divorcios y la destrucción de la familia. Pero ¿qué relación puede haber entre los cambios en la estructura familiar, las nuevas relaciones amorosas, la revolución sexual y las políticas de la identidad? Para Eberstadt el nexo de unión es el siguiente. En primer lugar, la pregunta por la identidad, «quién soy yo», es una pregunta universal que tradicionalmente era contestada a partir de la cultura y las relaciones orgánicas en las que uno crecía; los padres, abuelos, hermanos y primos nos situaban en unas coordenadas en las que reconocernos y en las que formar una identidad estable. La ruptura de esas relaciones ha tenido efectos en la construcción de la identidad del sujeto. Por ejemplo, analizando la encuesta de Elisabeth Marquardt y Norval Glenn, encontramos que los hijos de padres divorciados tenían tres veces más posibilidades de estar de acuerdo con la frase, «me sentía como una persona diferente con cada uno de mis padres» (Eberstadt, 2020, p. 43). Esa crisis de identidad es fuente de una generación miedosa que busca seguridades en la construcción de nuevas identidades que se imponen de manera reactiva. Según este mismo ensayo, por ejemplo, la causa del auge de un feminismo agresivo está en:

(U)na reacción protectora, terriblemente deformada pero profundamente sincera, frente un entorno hostil de riesgos elevados. En un mundo donde el sexo libre ha hecho que la compañía masculina sea más problemática que antes, algunas mujeres han adoptado la coloración protectora de las características masculinas: bravuconadas, lenguaje soez, beligerancia, actitudes desafiantes y, según sea necesario, promiscuidad 0 al menos la legitimación de esta (Eberstadt, 2020, p. 70-71).

En segundo lugar, y relacionado con lo anterior, hay una conexión de la vida moral con la sexualidad como no la hay en otras necesidades humanas, en la medida que esta dimensión pone en juego a varias personas y de manera íntima. Para acercarnos a este fenómeno desde las coordenadas de nuestro tiempo deberemos primero afrontar las explicaciones que provienen de las 
psicologías evolucionistas (Wilson, 2000; Barkow, Cosmides y Tooby, 1992) porque para muchos la psicología evolucionista ha arrancado el asunto de la moral de las manos de los filósofos y se la ha entregado a la biología (Haidt, 2013), y tampoco es cuestión de entregar un ámbito de la realidad humana tan fácilmente.

Estos autores entienden la dimensión moral desde categorías evolutivas. En este caso, los distintos sistemas para regular el comportamiento sexual de hombres y mujeres han funcionado como más eficientes a la hora de garantizar la reproducción en contextos ya desaparecidos, pero precisamente como dichos contextos han cambiado dichas normas resultan ahora disfuncionales (Earp y Savulescu, 2020).

Los sistemas morales triunfantes son como son porque ofrecen ventajas adaptativas, y no por ninguna razón metafísica ligada a concepciones esenciales de la naturaleza humana. Según este tipo de explicaciones, como la crianza exige unas energías y el compromiso del padre, por eso surgen normas morales que benefician aquellos comportamientos que tienen que ver con la fidelidad y la monogamia (Brandon, 2016). La explicación evolucionista supedita todo al mecanismo de selección natural y la adaptación al ambiente. Ciertamente, no siempre esta especie de darwinismo social es tan burdo y no pretende explicar así los comportamientos concretos, pero sí que lo pretende hacer con las dimensiones psicológicas fundamentales, que son aquellas que soportan los distintos códigos morales, y que son el fruto de los mecanismos evolutivos (Symons, 1989).
En primer lugar, el problema de este tipo de lectura de la vida moral es que resulta poco útil para el mundo educativo, bien porque no propone ningún criterio con el que decidir qué código moral resulta más conveniente transmitir, manteniéndose en un ámbito pura 0 principalmente descriptivo, o bien porque simplifica en exceso la vida humana al convertir los debates o dilemas morales en meros juegos del lenguaje que es incapaz de entender del todo. El psicólogo moral podrá analizar el comportamiento humano con una perspectiva no cualitativamente diferente a la de un etólogo que analiza empíricamente distintos comportamientos de cualquier animal social. En esta misma línea podemos englobar los estudios que, siguiendo estos paradigmas materialistas, pretenden ofrecer modelos de intervención educativa, 0 ventanas de oportunidad para la intervención a partir del estudio psicobiológico del desarrollo adolescente (Susman, Marceau, Dockray y Ram, 2019; Immordino-Yang, Darling-Hammond y Krone, 2019). Sin embargo, su explicación será siempre incompleta porque olvida la dimensión radical y cualitativamente distinta de la estructura personal del ser humano.

Por ejemplo, el ser humano, como el resto de especies que tienen una reproducción sexual, está sometido al proceso del apareamiento, pero los términos que ligamos a ese proceso en el ámbito humano, como el de fidelidad, por ejemplo, no pueden ser entendidos ni estudiados en la dimensión biológica porque, como dice Yela, «la diferencia radical entre el comportamiento animal y la conducta humana consiste en que aquel es siempre una relación entre el animal y su medio, y esta, una relación entre el 
hombre y su mundo» (Yela, 1996, p. 156). El mundo es una realidad a la que responder significativamente, una realidad a la que accedemos por un lenguaje significativo $\mathrm{y}$ en la búsqueda de sentido. La psicología evolucionista moderna no es capaz de entender esta dimensión porque no entra en sus esquemas, pero resulta imprescindible para los educadores porque forma parte de su horizonte de intervención. Scruton analiza, de manera interesante y sugerente, esta dificultad de la ciencia evolutiva para comprender en profundidad la naturaleza humana estudiando el fenómeno de la risa (Scruton, 2018).

Ese mundo de sentido es el ámbito en el que se mueve la moral sexual y la educación, pues es en el que nos hacemos cargo de esa dimensión. Por eso, el lenguaje que utilizamos para explicar las realidades sexuales es tan importante. Sin embargo, las filosofías de la sospecha han destruido cualquier tipo de pretensión de otorgar significado profundo a un lenguaje que pretenda fundar algún tipo de normatividad en este ámbito. A pesar de esto, necesitamos acercarnos a esta dimensión sexual, entendiendo que forma parte de la dimensión afectiva del ser humano y a través de un lenguaje que nos ayude a comprender las tendencias que subyacen a nuestros deseos, que los formen y que los dirijan a los fines que les son propios, en una dimensión que no es la puramente bioquímica, sino la personal.

Pero, el predominio de una moral meramente deontológica deja fuera, en muchas ocasiones, aquellos aspectos que no tienen que ver con el acuerdo entre partes. Esto es insuficiente para una educa- ción que pretenda ayudar en la búsqueda de sentido, en el hacerse cargo del mundo, también del mundo afectivo. En esta dimensión, una ética deontológica resulta insuficiente y necesitamos de una ética sustantiva. ¿Qué es una ética sustantiva? Entendemos por ética sustantiva aquella que tiene en cuenta que los seres humanos nos configuramos como personas que se autointerpretan, que necesitan un marco de referencia que explique quiénes somos y cómo podemos alcanzar una vida buena.

Configurarse como ser personal implica comprenderse como sujeto, consciente, con intenciones y propósitos e irreductible a sus propiedades biológicas, «me atrevería a sugerir que la persona tiene que ser entendida como una entidad emergente, enraizada en el ser humano, pero perteneciente a un orden distinto del biológico» (Scruton, 2018, p. 23). Pero situarse en un orden distinto al biológico no implica situarse al margen de lo biológico. Somos personas encarnadas en cuerpos sexuados y no simplemente tenemos esas propiedades como algo de lo que nos podemos independizar hasta convertirnos en espíritus puros. Aquí radica la crítica que Scruton realiza a lo que él llama feminismo kantiano, que no se toma en serio el carácter esencial de nuestra corporeidad sexuada. No podemos construir nuestra sexualidad como si el cuerpo fuese algo distinto a lo que somos en un dualismo desencarnado (Scruton, 2006). Nuestra corporalidad tiene exigencias en el plano biológico y también exigencias en la dimensión de nuestro ser personal.

Desde el punto de vista subjetivo la primera de estas exigencias estriba en la 
necesidad de entender que el placer sexual no es una mera respuesta fisiológica con sentido en sí mismo. El ámbito personal de la sexualidad está sometido a las reglas del trato entre personas y exige una apertura al otro como algo distinto a un mero objeto para nuestro disfrute. El placer en una sexualidad humana no es un placer sobre el otro, sino un placer en el otro (Scruton, 2018). Esta apertura al otro nos lleva a reconocer el tipo especialmente repulsivo que supone la violencia que se ejerce en el abuso sexual. Un tipo de violencia que implica tratar como objeto lo que es un sujeto. Cuando la sociedad pierde la repulsa al abuso sexual, pierde, en cierto sentido, la conciencia del ser personal.

La comprensión de la sexualidad humana supone también hacerse cargo de que no solo nuestra racionalidad es lo que nos abre a los otros, sino que también nuestra corporalidad es relacional. Esta dimensión relacional está sometida a una normatividad que colabora en nuestra creatividad y no la anula. Frente a las denuncias de la filosofía de la sospecha necesitamos comprender el valor de las normas morales, también en este ámbito, como formas de liberación (Reyero y Gil Cantero, 2019). Pondremos un ejemplo. Educados en el lenguaje de la sospecha existe un rechazo actual y creciente a los compromisos estables y duraderos y a las prácticas que institucionalizan lo que supuestamente es la libertad del sentimiento (Carter y Duncan, 2017), pero quizás el análisis alternativo que reivindicamos nos ayude a entender que es precisamente al revés. Chesterton lo explica muy bien. No es que la institucionalización de las relaciones humanas, como el matrimonio, resulte ser una mera y violenta imposición social para controlar un ciego impulso sin sentido, sino que más bien este vínculo es también la expresión de una profunda búsqueda de eternidad y permanencia en la relación que late en el corazón humano. Dice Chesterton:

No es cierto que todo amor joven nace libre de las tradiciones sobre unirse y prometerse, sobre lazos y firmas y sellos. Todo lo contrario, los enamorados se regodean en la más descabellada pedantería y precisión sobre estos asuntos. Hacen las cosas más extravagantes para hacer su amor legal e irrevocable: se tatúan uno a otro con promesas; esculpen rocas y robles con sus nombres y votos [...] El hecho alarmante, pero verdadero, es que la gente joven se muestra especialmente vehemente para establecer cadenas y ligaduras justo en el momento en el que piensan que son innecesarias. Desean el voto exactamente cuando no lo necesitan. He ahí algo que merece ser meditado (Chesterton, 1995, p. 227-228).

Parece claro que el deseo y el impulso que permite ligar la sexualidad al amor genera fuertes vínculos que van más allá de la satisfacción del apetito sexual, pues cuando el apetito se extingue, igual que el hambre después de comer, los vínculos se pueden acrecentar. Entendemos que eso es así, pues la sexualidad humana no se puede desligar de un tipo especial de comunicación y la comunicación nos vincula al otro, en este caso, de una manera que nos hace crecer en una relación de ayuda mutua, y reconocernos a nosotros mismos en los ojos del otro (Scruton, 2006).

Por último, si bien la sexualidad es una dimensión relacional abierta al otro y una forma de comunicación que compromete la 
intimidad, no todo tipo de relación sexual tiene las mismas exigencias. Dice Scruton que las relaciones heterosexuales resultan en este sentido más exigentes y también, precisamente por el significado de esa dificultad, complementarias, puesto que se dirigen «hacia un individuo cuyo género lo confina dentro de otro mundo» (Scruton, 2006, p. 283) (traducción del autor) $^{3}$. Dice Hadjadj hablando de su mujer y en este mismo sentido.

Cuanto más juntos vivimos, más descubre cada uno su soledad esencial y la alteridad irreductible del otro. Siffreine nunca está tan unida a mí como cuando yo reconozco que ella se me escapa por entero. La comunión consiste en resistir en esa distancia de lo íntimo, en la proximidad del misterio (Hadjadj, 2010, p. 177).

Pero, además, la relación de la sexualidad con la generación de una vida nueva sitúa también las relaciones heterosexuales en otro plano moral. En las relaciones sexuales entre hombre y mujer hay una vinculación misteriosa con un tercero que aún no existe, y que quizá no se espere, pero que es una posibilidad. "Uniendo sus carnes forman una sola carne distinta de ellos mismos» (Hadjadj, 2010, p. 178). La posibilidad de la paternidad es una señal del tipo de responsabilidad y madurez que la sexualidad exige.

\section{Conclusión}

Terminaremos con algunas reflexiones a modo de resumen.

1. Es imposible educar la sexualidad si no entramos en una vida intelectual abierta a la búsqueda de la verdad y de sen- tido. Esta apertura supone introducirnos en un lenguaje que no se conforme con la racionalidad científico-técnica para explicar la sexualidad humana y tampoco se fundamente en la sospecha de cualquier lenguaje que se comprometa con explicaciones teleológicas del ser humano. Solo un lenguaje con cierta densidad antropológica es capaz de ofrecer caminos educativos que nos permitan discernir qué deseos resultan convenientes y cuáles no, qué prácticas hacen justicia a nuestra naturaleza y cuáles la degradan.

2. Al ser una actividad entre personas está sometida a una específica normatividad cuya exigencia proviene del carácter íntimo que se pone en juego. Su educación tiene que ver con el cuidado del otro.

3. Si, como hemos mostrado, la intimidad está en juego cuando hablamos de sexualidad, no estaría de más recuperar viejas palabras, como pudor, entrega, pasión, lujuria o concupiscencia que nos permitan entender, por una parte, la belleza que encierra, y por otra, las emociones que se movilizan y los riesgos personales, y no solo de salud, que están en juego.

\section{Notas}

1 Parece probable que el autor no siempre pensase lo mismo en lo relativo a la revolución sexual de nuestro tiempo. En un interesante texto original de 1971, Ja. cinto Choza analiza la supresión del pudor en el ám. bito de la sexualidad como una propiedad de nuestra época, y como una manifestación de la supresión de la intimidad (Choza, 1990). No fue consciente entonces de hasta qué punto esa ruptura puede ser potenciada o causada por la revolución sexual. Sin embargo, el 
modo en el que los adolescentes actuales se exponen en las redes sociales y el mundo «transparente» y de sobreexposición que estamos creando, cuestiona la idea de que las consecuencias sobre la intimidad de esa revolución sean siempre tan positivas. Recorda. mos aquí que Choza da al pudor una definición positi. va, incluso necesaria, para entender la intimidad y la propia vida personal: «El pudor es el hábito y la ten. dencia a mantener la posesión de la propia intimidad desde la instancia más radical de la persona (el yo), y a mantener dicha intimidad en el estado de máxima perfección posible, con vistas a una entrega por la cual se trasciende la soledad y se autoperfecciona el suje. to» (Choza, 1990, p. 28).

${ }^{2}$ Hay una interesante discusión de Roger Scruton con Martha Nussbaum a raíz de este punto que puede leerse resumida en (Reyero, 2020).

${ }^{3}$ La revista española de pedagogía se publica en espa. ñol y en inglés. Por este motivo, sigue el criterio, cuan. do se citan textos ajenos, de acudir a los originales que están escritos en esas lenguas y de poner su traducción oficial, cuando tal texto se haya editado también en el otro idioma. En caso de que no se haya producido esa traducción oficial, el texto citado se ofrecerá a los lec. tores traducido o por el autor del artículo (señalándose que la traducción es del autor del artículo), o por el traductor jurado contratado por la revista.

\section{Referencias bibliográficas}

Barkow, J. H., Cosmides, L. y Tooby, J. (Eds.) (1992). The adapted mind: Evolutionary psychology and the generation of culture [La mente adaptada: la psicología evolutiva y la generación de cultura]. Oxford University Press.

Bloom, A. (2008). The closing of the american mind [El cierre de la mente moderna]. Simon and Schuster.

Brandon, M. (2016). Monogamy and nonmonogamy: Evolutionary considerations and treatment challenges [Monogamia y no monogamia: Consideraciones evolutivas y dificultades para el tratamiento]. Sexual medicine reviews, 4 (4), 343-352. https://doi.org/10.1016/j.sxmr.2016.05.005

Carter, J. y Duncan, S. (2017). Wedding paradoxes: individualized conformity and the 'perfect day' $[\mathrm{Pa}-$ radojas de las bodas: conformidad individualizada y el «día perfecto»]. The sociological review, 65 (1), 3-20. https://doi.org/10.1111/1467-954X.12366
Chesterton, G. K. (1995). El amor o la fuerza del sino. Rialp.

Choza, J. (1990). La supresión del pudor y otros ensayos. EUNSA.

Choza, J. (2020). El sexo de los ángeles. Sexo y género desde las bacterias a los robots. Editorial Thémata.

Collins, C., Alagiri, P., Summers, T. y Morin, S. F. (2002). Abstinence only us. comprehensive sex education: What are the arguments. What is the evidence. AIDS Research Institute, University of California. https://bit.ly/2JAOtHV

Earp, B. D. y Savulescu, J. (2020). Evolved Fragility [Fragilidad evolucionada]. En Love is the drug (pp. 101-109). Manchester University Press.

Eberstadt, M. (2020). Gritos primigenios: Cómo la revolución sexual creó las politicas de identidad. Ediciones Rialp.

Fine, M. y McClelland, S. (2006). Sexuality education and desire: Still missing after all these years [La educación sexual y el deseo: aún ausente después de todos estos años]. Harvard Educational Review, 76 (3), 297-338. https://doi.org/10.17763/ haer.76.3.w5042g23122n6703

Fine, M. (1988). Sexuality, schooling, and adolescent females: The missing discourse of desire. Harvard Educational Review, 58 (1), 29-54. https://doi. org/10.17763/haer.58.1.u0468k1v2n2n8242

Giroux, H. (2013). La pedagogía crítica en tiempos oscuros. Praxis educativa, 17 (2), 13-26.

Hadjadj, F. (2010). La profundidad de los sexos. Por una mística de la carne. Nuevo Inicio.

Haidt, J. (2013). Moral psychology for the twenty-first century [Psicología moral para el siglo xxI]. Journal of Moral Education, 42 (3), 281-297. https:// doi.org/10.1080/03057240.2013.817327

Harden, K. P. (2014). A sex-positive framework for research on adolescent sexuality [Un marco sexual positivo para la investigación sobre la sexualidad de los adolescents]. Perspectives on Psychological Science, 9 (5), 455-469. https://doi. org/10.1177/1745691614535934

Immordino-Yang, M. H., Darling-Hammond, L. y Krone, C. R. (2019). Nurturing nature: How brain development is inherently social and emotional, and what this means for education [Nutrir la naturaleza: Cómo el desarrollo del cerebro es inherentemente social y emocional, y lo que esto significa para la educación]. Educational Psychologist, 54 
(3), 185-204. https://doi.org/10.1080/00461520.20 19.1633924

Irvine, J. M. (2004). Talk about sex: The battles over sex education in the United States [Hablar de sexo: Las batallas en torno a la educación sexual en los Estados Unidos]. University of California Press.

MacIntyre, A. (2001). Animales racionales y dependientes: por qué los seres humanos necesitamos las virtudes. Paidós.

Pieper, J. (2005). Introducción a Tomás de Aquino. Doce lecciones. Rialp.

Reyero D. (2020). Teoría mimética y educación sexual. Contra los géneros en conflicto. Xiphias Gladius. Revista Interdisciplinar de Teoría Mimética, 3. https://doi.org/10.32466/eufv-xg.2020.3.635.121-138

Reyero, D. y Gil Cantero, F. (2019). La educación que limita es la que libera $\mid$ Education that limits is education that frees. revista española de pedagogía, 77 (273), 213-228. https://doi.org/10.22550/ REP77-2-2019-01

Rorty, R. (1990). Educación sin dogma. Facetas, 2, 44-47.

Santelli, J. S., Kantor, L. M., Grilo, S. A., Speizer, I. S., Lindberg, L. D., Heitel, J., Schalet, A. T., Lyon, M. E., Mason-Jones, A. J., McGovern, T., Heck, C. J., Rogers, J. y Ott, M. A. (2017). Abstinence-only-until-marriage: An updated review of US policies and programs and their impact [Abstinencia hasta el matrimonio: una revisión actualizada de las políticas y programas de EE.UU. y su impacto]. Journal of Adolescent Health, 61 (3), 273-280.

Scruton, R. (2006). Sexual desire: A philosophical investigation [El deseo sexual: una investigación filosófica]. Continuum.

Scruton, R. (2017). Bebo luego existo. Rialp.

Scruton, R. (2018). Sobre la naturaleza humana. Rialp. Symons, D. (1989). A critique of Darwinian anthropology [Una crítica a la antropología darwiniana]. Ethology and Sociobiology, 10 (1-3), 131-144. https://doi.org/10.1016/0162-3095(89)90016-2

Spaemann, R. (2003). ¿Es la emancipación un objetivo de la educación? En Limites. Acerca de la dimensión ética del actuar (pp. 453-465). EIUNSA.

Spaemann, R. (2017). Prólogo a la edición alemana. En G. Kuby (2017), La revolución sexual global. La destrucción de la libertad en nombre de la libertad (pp. 27-29). Didaskalos.

Susman, E. J., Marceau, K., Dockray, S. y Ram, N. (2019). Interdisciplinary work is essential for re- search on puberty: Complexity and dynamism in action [El trabajo interdisciplinario es esencial para el studio de la pubertad: complejidad y dinamismo en acción]. Journal of Research on Adolescence, 29 (1), 115-132. https://doi.org/10.1111/ jora. 12420

Tolman, D. L. (2012). Female adolescents, sexual empowerment and desire: A missing discourse of gender inequity [Las adolescentes, el empoderamiento sexual y el deseo: un debate pendiente sobre la desigualdad de género]. Sex Roles, 66, 746-757. https://doi.org/10.1007/s11199-012-0122-x

Tolman, D. L. y McClelland, S. I. (2011). Normative sexuality development in adolescence: A decade in review, 2000-2009 [Desarrollo de la sexualidad normativa en la adolescencia: una década en revisión, 2000-2009]. Journal of Research on Adolescence, 21 (1), 242-255. https://doi.org/10.1111/ j.1532-7795.2010.00726.x

Wilson, E. 0 (2000). Sociobiology: The new synthesis [Sociobiología: la nueva sintesis]. Harvard University

Yela, M. (1996). Comportamiento animal y conducta humana. Psicothema, 8, 149-163.

\section{Biografía del autor}

David Reyero García. Doctor en ciencias de la educación por la Universidad Complutense de Madrid y Profesor Titular en dicha universidad. Actualmente es Subdirector del Departamento de Estudios Educativos, Codirector del Grupo de Investigación en Antropología y Filosofía de la Educación (GIAFE) y Editor Adjunto de la Revista de Educación del Ministerio de Educación. Sus publicaciones abordan aspectos relacionados con la epistemología propia del conocimiento pedagógico, las nuevas tecnologías, la educación cívica, la política y economía de la educación o los fines morales de la misma.

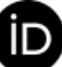

https://orcid.org/0000-0002-9047-532X 


\section{Is there a sexual education that is an expression of a cultivated intelligence?}

\section{¿Cabe una educación sexual que sea expresión de una inteligencia cultivada?}

David REYERO, PhD. Associate Professor. Universidad Complutense de Madrid (reyero@ucm.es).

\section{Abstract:}

This article aims to show how sex education today responds to the dominance that philosophies of suspicion have achieved over intellectual life, a dominance that hinders a normative judgment of human sexuality. Any attempt at regulation is suspected of concealing some kind of domination by some over others. The lack of regulations makes meaningful education about sexuality impossible and only allows for education that is superficial, instrumental, and limits itself to managing unwanted consequences such as teenage pregnancies and sexually transmitted infections. This work shows that there is another way of understanding intellectual life that is linked to moral life, open to the truth, and not just dedicated to denunciation. Thinking well involves living within a tradition and on the path of a good life. A concept of a good life supposes a teleological understanding of the human con- dition and the necessary cultivation of virtues in order to remain within it. In this dimension, sexuality has a meaning that is not merely biological but also relational, generative, and communicative, and it is subject to rules that derive from our personal character and sexuality's relationship with intimacy.

Keywords: sex education, thinking, affective education, moral education.

\section{Resumen:}

El presente texto pretende mostrar cómo la educación sexual en la actualidad responde al dominio que las filosofías de la sospecha han conseguido sobre la vida intelectual. Este dominio dificulta un juicio normativo sobre la sexualidad humana. Cualquier intento de normatividad es sospechoso de esconder algún tipo de dominación de unos sobre otros.

Revision accepted: 2020-09-28.

This is the English version of an article originally printed in Spanish in issue 278 of the revista española de pedagogía. For this reason, the abbreviation EV has been added to the page numbers. Please, cite this article as follows: Reyero, D. (2021). ¿Cabe una educación sexual que sea expresión de una inteligencia cultivada? | Is there a sexual education that is an expression of a cultivated intelligence? Revista Española de Pedagogía, 79 (278), 115-129. https://doi.org/10.22550/REP79-1-2021-05

https://revistadepedagogia.org/ 
La ausencia de normatividad imposibilita una educación de la sexualidad significativa y solo permite una educación superficial, instrumental y limitada al manejo de las consecuencias no deseadas: embarazos adolescentes, enfermedades de transmisión sexual, etc. Mostramos la existencia de otra forma de entender la vida intelectual ligada a la vida moral, abierta a la verdad y no solo dedicada a la denuncia. Pensar bien implica vivir en el seno de una tradición y en el camino de la vida buena. Una idea de vida buena supone la comprensión teleológica de la condición humana y el necesario cultivo de unas virtudes para mantenerse en ella. En esa dimensión, la sexualidad tiene un sentido no meramente biológico sino relacional, generativo y comunicativo, y está sometida a unas reglas fruto de nuestro carácter personal y de la relación que la sexualidad tiene con la intimidad.

Descriptores: educación sexual, pensamiento, educación de la afectividad, educación moral.

\section{The intellectual life}

In 1975, Robert Spaemann wrote an interesting work called Is emancipation an educational goal? The article was provocative as the concept of emancipation was in vogue at the time and still is when discussing the objectives of education. His surprising and quick answer is a resounding no, although we must now qualify it since Spaemann is certainly not proposing a pedagogy of command, nor an ideal of a subjugated human being. The enemy Spaemann confronts is a specific way of understanding thought that is ultimately based on suspicion and mistrust. How we understand human thought's relationship with education and the different dimensions of the person will depend on how we understand its function. For ideologues of emancipation, everything must start from a slow process of becoming self-aware of those aspects of ourselves that react to interests we are not the masters of and which result from a tradition. This tradition offers us meaning, a way of life, and a language with which we can explain ourselves and the reality that surrounds us to ourselves. But this language also conceals or justifies asymmetric power relationships and unequal power distributions. According to these philosophies of suspicion, it appears that human beings, even when they do not know it, are slaves as they are embedded in relationships they have not chosen and from which they do not always have the possibility of emancipation. Consequently, any interest is suspicious and it is the task of thought to uncover what is hidden from view. This way of understanding thought has also been expressed in the field of pedagogy where it is known as critical pedagogy. For critical pedagogy, the function of the type of thought we know as pedagogy is not the design of strategies for transmitting established knowledge. Instead, "critical pedagogy illuminates the relationships among knowledge, authority, and power. For instance, it raises questions regarding who has control over the conditions for the production of knowledge" (Giroux, 2013, p. 28). 
Critical pedagogy involves suspicion of any asymmetric relationship based on authority or tradition. Its objective is what is known as radical democracy. This democracy is the outcome of a prior pedagogical undertaking that deconstructs any ideology that shapes us, since "ideologies are not just a constellation of ideas, stereotypes, and modes of common sense; they also represent specific forms of knowledge and beliefs rooted in strong emotional investments. Such attachments need to be understood, analysed, and deconstructed, often not simply as a form of uncomprehending knowledge but as an active refusal to know and the refusal 'to acknowledge one's own implication' with such attachments" (Giroux, 2013, p. 35). Therefore, any ideology produces within itself some type of oppression against anyone who does not want to or cannot submit to its categories and to the norms these bring with them.

The role of thought is therefore to reveal, or rather denounce, because behind meaning there is always a hidden interest. This way of directing the cultivation of intelligence is clearly insufficient, even though it might seem attractive, as it would derive from the recognition that what is of value in thought lies in its capacity for revealing/expose errors, abuse, or lies rather than in revealing/ exposing what is of value, "interesting", good, or true.

The rationale of this way of understanding thought, and consequently the value of the intellectual life, rests on two errors. The first is a certain misunder- standing of the limited character of human life and thought. In the face of this limitation, the allure of novelty offers an escape from the boredom or weariness that so often affects the everyday. In effect, thinking is not just the logical concatenation of ideas and arguments, but instead involves entering into a tradition with specific practices and certain demands that also affect the way of life and habits that keep us in it. Thought, therefore, has a relationship with this second nature, which we call moral life, and not just with logic. This second nature is laboriously built and is always supported by a meaningful language whose veracity cannot be discovered if we do live in it.

This is the sense of the critique that, for example, MacIntyre (1999) makes of Richard Rorty's concept of critical education. For Rorty, developed thought, critical thought, is thought that is capable of irony once it has been socialised in a way of seeing reality. To be fair, it is admittedly necessary to recognise that, According to Rorty, there is no possibility of thinking critically without first acquiring a certain type of language and comprehension of the world, something he links to socialisation (Rorty, 1989). But for Rorty, what is characteristic of the human being is the possibility of casting doubt on this ultimate thought, creatively ironising with it, with all of the consequences this entails.

When facing this dominant way of understanding intellectual life, authors such as MacIntyre offer alternatives. Essentially the human being is a profoundly 
dependent being whose telos is to manage to become independent, or have independent thought, or its own critical thought. For MacIntyre, what is unusual about this journey to independence is that it can only be done from dependency. This might seem to match with the need for socialisation in Rorty, but they are not the same. For MacIntyre, dependence is a precondition for the possibility of independent thought, which he calls "independent practical reasoning". We can become independent thanks to others, not despite them. This has an important implication; suspicion when confronting received ideas is not the objective of human life and is not even something essentially desirable. MacIntyre (1999) states that we must have very good reasons to oppose those who love us and have taught us to be who we are. And while doing this is sometimes necessary, and learning this difficult lesson is an important part of education, it is always necessary to have very good reasons when doing it.

MacIntyre underlines the importance of intellectual training within a tradition, the idea that we always learn what we are thanks to others, and the idea that tradition is something qualitatively different to socialisation. He chooses to explore what he calls virtues of dependence, the type of attitudes that enable a better social life. Truthfulness stands out among the necessary virtues. Here it is important to recall that for Rorty any discourse on truthfulness is an inconvenience while for MacIntyre it is fundamental, not just for thought, understood as pure cognition, but also for the formation of moral judgement.
For MacIntyre there are three attitudes that harm the necessary virtue of truthfulness: "A first type of offense against truthfulness then consists in unjustly preventing others from learning what they need to learn, and a second type consist in concealing from view the nature of our relationships to those others" (MacIntyre, 1999, p. 151). The third aggression MacIntyre discusses is precisely Rorty's irony, which he identifies with the sophistical attitude that is unable to commit to a final vocabulary. Why? Firstly, because a final vocabulary is not a sort of superficial socialisation of customs that enables us to appear before others and be recognised, but rather also a vocabulary that commits us, explains us, enables us to understand the human world, and allows it to function. How would a society work if we could not really trust one another, or if we did not believe that, at least to a large extent, we understand the same thing about what is just, good, and true? How could we even shop in the supermarket without trusting in the truthfulness of what we are told? Should we mistrust someone who sells us a litre of milk when they say they are selling a litre of milk? Why attend class and believe our teachers if we do not think that what they tell us is essentially true? For MacIntyre, ironic detachment is a form of cynicism that would make social life unliveable. If ironic detachment is genuine and not just a pose, if it truly questions the foundations on which we have been socialised, we would be at the mercy of the strongest, the most powerful, those who have the most resources for imposing their agenda. 
For MacIntyre, criticism is not always necessary or of value for advancing in knowledge. MacIntyre criticises the idea of placing the foundations of the intellectual life in the field of mistrust and doubt. His essential argument relates to progress through virtue. For example: if we ask ourselves why we would not betray a friend if it benefits us, this means that we are still at a very basic level of comprehension of what friendship means. A person who progresses in the cultivation of this virtue will reach a point where he can no longer ask himself this question, and this inability is an achievement that gives a better and more perfect thought and situates him before the possibility of having better judgement with regards to the practice of friendship.

In summary, the cultivated intellectual life is not that which is born from mistrust or questioning, because questioning our interests, their history and genealogy is insufficient and is not even useful. We must have interests and these interests are achieved in another way. A fully human life is a life with interests (Spaemann, 2003). The true intellectual life is one that is born from insight and improvement in the search for truly valuable goods or practices, and this thought requires practice and full immersion in a particular type of life, whether it be scientific, philosophical, humanist, or religious. Thinking well involves living in a certain way and not just reasoning in a given way. From this perspective, we can understand that phenomena such as communion, have the same sense and the same mentality, they are the consequence of a given intellectual life marked by experience of shared knowledge that is the fruit of a same tradition. Knowledge

per connaturalitatem, as a man recognizes his beloved or what is his own. The stranger does not understand, or misunderstands, but one who is allied with another in love and congeniality knows immediately, and with absolute certainty, what is meant in a fragment of a letter or a dimly heard call. (Pieper, 2011)

This idea leads us to the second error of suspicious thought. Models derived from suspicion paradoxically result in the exaltation of the apparently creative or original and of identity and sentimentality. Identitarian postmodernism is the inevitable child of rationalist modernity which is the mother of the philosophies of suspicion.

As we have just seen, modernity, the origin of the critical thinking being discussed here, tried to break its bonds with tradition and with a way of thinking that derives from a profound teleological view of the human being. This break has been a tough blow for the formation of thought, which now has no criteria other than its own originality and its capacity for seduction. The validity of thought now lies in the creativity it displays or in its capacity to entertain or dazzle, not in its power to unravel human nature. It is in this very ordinary sense that, for example, adolescents are captivated by fashionable series that offer channels for desire behind an attractive façade while at the same concealing important aspects of human nature. 
If all that remains is suspicion, we can only turn to the experience of our own desire, which, without reliable criteria for judgment or reference, is the only thing that appears to be truly real. What do I do with my desire, which feels like an ache constantly demanding my attention? In the absence of other substantive criteria, all that remains is to prioritise this desire fully, and as long as it does not affect others, this seems to be the most rational course. Consequently, the intellectual life is a life that accompanies or finds reasons to justify or legitimise this desire.

\section{The formation of human sexu- ality and tradition}

There is a strong relationship between the intellectual life that stems from philosophies of suspicion and the current conception of sexuality and its formation. Education, in the contemporary world, as described above, only concerns itself with the most negative consequences of sexuality: teenage pregnancy, sexually transmitted infections, consideration of different sexual identities and orientations, or the patriarchy, which refers to the supposed unequal distribution of power between men and women. In any case, we can study sexuality so long as we do not enter into the question of proper or improper behaviour, terminology of which we should be suspicious. But talking about sexuality at school has never been simple, here or anywhere. This is because the debate about what school can or should say about this matter is marked by deep-rooted and rival anthropological conceptions (Irvine, 2004).
For example, contrasting the two most classical currents of sex education in the United States, "Abstinence Only" and "Comprehensive Sex Education", reveals very different conceptions of value and morality. For the former, full sexual relations outside of wedlock are immoral per $s e$, and information about ways of avoiding pregnancy can be counterproductive as it encourages adolescents to do sexual activities for which they are not psychologically ready, which are morally reprehensible, and which by their very nature can lead to everything their opponents also want to avoid, sexually transmitted infections and teenage pregnancies, with the consequent fall into poverty, marginalisation, etc.

In contrast, the supporters of what Americans call comprehensive sex education maintain that it is not true that information about different methods of contraception encourages earlier sexual activity. They also argue that there is a right to information about sex and to education in the practices that permit "safe sex". Their ultimate reasoning is that we live in a plural society and we do not all share the same fundamental anthropological concepts, so what we have to solve is not a moral problem as such - although for some people it is - but a public health issue on which we all agree (Fine, 1988; Fine \& McClelland, 2006; Collins et al., 2002; Santelli et al., 2017). Within this current there is another series of studies that set out to go beyond the perspective centred on adolescent sex as a risk activity to focus on a positive consideration, according to them, of adolescent 
sexuality, emphasising its importance as an individualising force and a space for confronting dominant models in relationships between men and women (Tolman, 2012; Tolman, \& McClelland, 2011; Harden, 2014). But however positively they regard their approach, their analysis is supported on a sociological, or at most psychological, dimension of the phenomenon of human sexuality. They never enter into the anthropological dimension or the dimension of meaning.

The problem with these two methods is that both encounter the same obstacle, which is essentially intellectual and which we could call "puritanism" or simplicity. The term "puritanism" here has the same sense Roger Scruton gives it: for him, puritanism is a form of laziness that when faced with a complex problem attempts to overcome it by the easiest route (Scruton, 2009, p. 139). As education in affection, of which education about sexual desire is a part, is a complex matter that requires diligent and sustained work, yet people apply the easy solution of pure negation of desire or treat the most disagreeable and inconvenient aspects of its expression. Those who argue for the route of the simple option of abstinence are lazy if they focus on this point alone, as they set out to solve a problem without helping to cultivate the virtues that are necessary to channel affection, nor do they show the beauty of achieving it. The actual results thus seem to be ineffective (Santelli et al., 2017).

On the other hand, those who set out to resolve the matter of sex education sim- ply through technical control of unwanted consequences, through information about condoms and "safe practices", also display laziness, as they voluntarily eschew entering into the meaning of sexual desire for the human being and so they break any type of bond with the meanings sexuality has traditionally had. Their proposal is also lazy in that it leaves adolescents without reference points for understanding their desire and how to confront it.

Both move among the remains of an understanding in a world where the link between sexuality, stability, love, and reproduction has been broken. A world where easy access to contraceptive methods, and a complete availability of sex online prevail.

To deal with the subject of human sexuality and how cultivating the intellectual life can help with its formation, it is necessary to understand that there is a way of approaching this dimension of the person that does not derive from the pedagogy of suspicion, nor from acknowledgement of the existence of a desire that there is no reason to repress so long as it is done with sole limit of not causing harm to others. Instead it derives from the capacity to learn attentively from history and experience while seeking the meaning of our affective sexual reality. What can we learn from this observation?

Firstly, sexuality is an important dimension that must be approached without the purely defensive and fearful attitude with which it has perhaps been approached educationally in other periods, 
but also without trivialising it. Sexuality is an important part of processes of individualisation and human growth and is, in a way, sacred for humans since it combines two dimensions: intimacy and the creation of new life.

That sexuality is related to intimacy is evident just from observing reactions to sexual violence. This inspires greater revulsion than other types of violence because people see sexual violence as an attack on intimacy that profoundly affects those who are subjected to it. Without this relationship between sexuality and intimacy it would be difficult to explain why a simple look can cause more backlash than a fight over a sporting contest in a school playground. Moreover, the relationship between sexuality and reproduction is evident, even if people try to deny it. No matter how much people talk of safe sex nowadays as a practice that cannot lead to pregnancy, it remains true that we all come from a sexual relationship. The constant regulation of sexuality that different cultures have exercised originates in this connection between intimacy and reproduction. This "sanctity" of human sexuality is apparent in the mass of norms and rituals that have historically regulated its functioning. No bodily function is more subjected to institutionalisation than sexuality. As Spaemann says, this essential character of human sexuality, that distinguishes it from other ways of satisfying needs, "is subjected to certain rules of humanisation and has the privilege that derives from its institutionalisation" (Spaemann, 2017, p. 29). Institutionalisation is the human way of marking practices that are socially important.

However, we cannot ignore the fact that in the last half century there has been an intensive and constant process of privatisation or deinstitutionalisation of sex, running in parallel with an ongoing separation of sexuality and reproduction. This separation or break has increased thanks to birth control methods and the revolution that these have entailed for human life (Choza, 2020, p. 138-141). For Choza, this break between sex and reproduction liberates sex, which no longer has negative social consequences so long as the will of the other is respected. Consequently, for him the old moral architecture built around the connection between sex and reproduction now lacks meaning and is hard to transmit. He understands that on the whole there is a gain in this process (Choza, 2020, p.273-276).

Choza anticipates, perhaps prematurely, that the break between reproduction and sexuality will not have effects on intimacy. However, this anticipation might not be true, as the internet and other current social phenomena are starting to show us ${ }^{1}$.

In a recent essay, Mary Eberstadt (2019) claims that the problems resulting from the rise of identity politics, and which have led to a great degree of polarisation and violent outbursts, including within academic institutions, have their ultimate roots in the global sexual revolution and the rupture this has involved for the family. Her essay refers to Alan Bloom's famous book 
The closing of the American mind (2008). In this book, Bloom complains about the relativistic path higher education is following. One of the causes Bloom identified for this relativistic trend relates to the increase in divorce and destruction of the family. But what relationship might there be between changes in family structure, new romantic relationships, the sexual revolution, and identity politics? For Eberstadt, the connecting link is the following. Firstly, the question about identity — who am I? - is a universal question that was traditionally answered on the basis of the culture and organic relationships in which people were raised; parents, grandparents, siblings, and cousins located us in coordinates in which we could recognise ourselves and form a stable identity. The breakdown of these relationships has had effects on the construction of the subject's identity. For example, analysing the survey by Elisabeth Marquardt and Norval Glenn, we find that children of divorced parents were three times as likely to agree with the phrase, "I felt like a different person with each of my parents, even when I was a little kid" (Eberstadt, 2019, p.25). This identity crisis is the source of a fearful generation that seeks certainty in the construction of new identities that are imposed reactively. According to this same essay, for example, the cause of the rise of an aggressive feminism:

is instead an ultimately self-defeating but profoundly felt protective reaction to an environment of heightened risk. In a world where laissez-faire sex has made male companionship more peripatetic than before, some women will take on the protective coloration of male characteristics- blustering, cursing, belligerence, defiance, and also, as needed, promiscuity, or at least shout-outs thereto. (Eberstadt, 2019, p.56)

Secondly, and relating to the above, there is a connection between moral life and sexuality that is not present with other human needs since this dimension brings into play various people and does so intimately. To approach this phenomenon from the coordinates of our time, we must first consider explanations from evolutionary psychology (Wilson, 2000; Barkow, Jerome, Cosmides, \& Tooby, 1992) because for many people, evolutionary psychology has taken the question of morality out of the hands of philosophers and given it to biology (Haidt, 2013), and an area of human reality should not be abandoned so easily.

These authors understand the moral dimension from evolutionary categories. In this setting, the different systems for regulating the sexual behaviour of men and women have worked most efficiently when guaranteeing reproduction in contexts that have now disappeared, but precisely because these contexts have changed, these rules are now dysfunctional (Earp \& Savulescu, 2020).

Triumphant moral systems are how they are because they offer adaptive advantages, and not for any metaphysical reason linked to essential conceptions of human nature. According to this type of explanation, as raising children demands energy and the commitment of the father, moral norms appear that benefit behaviours that are connected to faithfulness 
and monogamy (Brandon, 2016). The evolutionary explanation subordinates everything to the mechanism of natural selection and adaptation to the environment. In fact, this sort of social Darwinism is not always so crude and it does not set out to explain specific behaviours in this way, but it does set out to do so with fundamental psychological dimensions, which are those that support the different moral codes, and which are the outcome of evolutionary mechanisms (Symons, 1989).

In the first place, the problem with this type of reading of moral life is that it is of little use in the world of education, either because it does not propose any criteria for deciding which moral code is most appropriate to transmit, limiting itself to a pure or primarily descriptive field, or because it simplifies human life excessively by turning moral debates or dilemmas into mere linguistic games that are incapable of understanding the whole. The moral psychologist can analyse human behaviour with a perspective that is not qualitatively different from that of an ethologist who empirically analyses different behaviour in any social animal. On the same lines we can include studies that, following these materialist paradigms, set out to offer educational intervention models or windows of opportunity for intervention based on psycho-biological study of adolescent development (Susman, Marceau, Dockray, \& Ram, 2019; Immordino-Yang, Darling-Hammond, \& Krone, 2019). Nonetheless, the explanations they provide will always be incomplete because they neglect the radical and qualitatively dis- tinct dimension of the personal structure of the human being.

For example, the human being, like other species that use sexual reproduction, is subject to the process of pairing, but the terms we connect to this process in the human sphere such as faithfulness, for example, cannot be understood or studied in the biological dimension. As Yela observes, "the radical difference between animal behaviour and human behaviour is that the former is always a relationship between the animal and its environment, while the latter is a relationship between the human being and its world" (Yela, 1996, p. 156). The world is a reality to which we must respond meaningfully, a reality we access through meaningful language and in a search for meaning. Modern evolutionary psychology is not capable of understanding this dimension because it does not enter into its schemes, but it is vital for educators, because it forms part of their area of intervention. Scruton provides an interesting and stimulating analysis of the problem evolutionary science encounters when understanding human nature in depth by studying the phenomenon of laughter (Scruton, 2019).

This world of meaning is the sphere where sexual morals and education move, as it is in it that we take charge of this dimension. This is why the language we use to explain sexual realities is so important. Nonetheless, philosophies of suspicion have destroyed any type of effort to give a profound meaning to a language that intends to establish some type of set of norms in this area. Despite this, it is 
necessary to approach this sexual dimension with the understanding that it forms part of the affective dimension of the human being, and to do so through a language that helps understand the tendencies that underlie desires, that shape them and direct them to their own ends in a dimension that is not purely biochemical but instead personal.

However, the dominance of a merely deontological morality often omits those aspects that do not relate to agreement between parties. This is insufficient for an education that aims to help in the search for meaning, in the process of taking charge of the world including the affective world. A deontological ethics is insufficient in this dimension and so a substantive ethics is needed. But what is a substantive ethics? A substantive ethics is that sort which takes into account the fact that as human beings we configure ourselves as people who interpret ourselves, who need a framework of reference to explain who we are and how we can achieve a good life.

Configuring oneself as a personal being involves understanding oneself as a subject that is conscious, has aims and intentions, and cannot be reduced to its biological properties, "I would suggest that we understand the person as an emergent entity, rooted in the human being but belonging to another order of explanation than that explored by biology" (Scruton, 2019, p. 30). But situating ourselves in an order of explanation other than biology does not mean separating ourselves from the biological. We are people who are incarnated in sexed bodies; we do not simply have these properties as something from which we can free ourselves until we become pure spirits. This is the basis of Scruton's criticism of what he calls Kantian feminism, which does not take seriously the essential character of our sexed corporeality. We cannot construct our sexuality as though the body were something other than what we are in a disembodied dualism (Scruton, 2006). Our corporeality has demands at the biological level and also has demands in the dimension of our personal being.

From the subjective point of view, the first of these demands revolves around the need to understand that sexual pleasure is not merely an inherently meaningful physiological response. The personal sphere of sexuality is subject to the rules of relations between people and demands an openness to the other as something other than a mere object for our enjoyment. Pleasure in a human sexuality is not pleasure in the other but pleasure with the other (Scruton, 2019). This openness to the other leads us to recognise the especially repugnant form of violence involved in sexual abuse. A type of violence that involves treating as an object someone who is a subject. When society loses its repulsion at sexual abuse, in a certain way, it loses the conscience of the personal being.

Understanding human sexuality also involves accepting that it is not just our rationality that opens us to others, but that our corporeality is also relational. This relational dimension is subjected to a normativity that cooperates in our creativity and does not cancel it. When facing the 
denunciations of the philosophy of suspicion we need to understand the value of moral norms as forms of liberation in this field as well (Reyero, Gil Cantero, 2019). Here is an example: Educated in the language of suspicion there is a current and growing rejection of stable and lasting commitments and the practices that institutionalise what is supposedly the freedom of feeling (Carter \& Duncan, 2017), but the alternative analysis we assert will perhaps help us to understand that it is precisely the opposite. Chesterton explains this very well. It is not that the institutionalisation of human relationships such as marriage is merely a violent social imposition to control a blind impulse without meaning, but rather this bond is also the expression of a profound search for eternity and permanence in the relationship that beats in the human heart. Chesterton says:

It is not the fact that every young love is born free of traditions about binding and promising, about bonds and signatures and seals. On the contrary, lovers wallow in the wildest pedantry and precision about these matters. They do the craziest things to make their love legal and irrevocable. They tattoo each other with promises; they cut into rocks and oaks with their names and vows; ... The startling but quite solid fact is that young people are especially fierce in making fetters and final ties at the very moment when they think them unnecessary. The time when they want the vow is exactly the time when they do not need it. That is worth thinking about. (Chesterton, 2005, p. 177)

It seems clear that desire and the impulse that makes it possible to link sexuality to love generates strong bonds that go beyond satisfying sexual appetites, since bonds can be strengthened when the appetite is extinguished like in the case of hunger after eating. We understand that this is so because human sexuality cannot be separated from a special type of communication and, in this case, communication links us to the other in a way that makes us grow in a relationship of mutual assistance, and recognise ourselves in the eyes of the other (Scruton, 2006).

Finally, although sexuality is a relational dimension that is open to the other and a form of communication that involves intimacy, not all types of sexual relationships have the same demands. Scruton claims that heterosexual relationships are more demanding in this sense and also, precisely because of the meaning of this difficulty, they are complementary since they are directed "towards an individual whose gender confines him within another world" (Scruton, 2006 p. 283)². Hadjadj, speaking on the same lines about his wife, states:

The closer we live, the more each of us discovers our own essential solitude and the irrepressible alterity of the other. Siffreine is never as united to me as when I recognise that she escapes from me fully. Communion involves enduring in that distance from intimacy, in the proximity of the mystery. (Hadjadj, 2010, p. 177)

But, in addition, the connection between sexuality and the creation of new life also places heterosexual relationships on another moral plane. In sexual relations between man and woman, there is 
a mysterious link with a third being that does not yet exist, and perhaps is not hoped for, but which is a possibility. "By uniting their flesh they form a single flesh different from themselves" (Hadjadj, 2010, p. 178). The possibility of parenthood is a sign of the type of responsibility and maturity that sexuality demands.

\section{Conclusion}

This article ends with some reflections that act as a summary.

1. It is impossible to educate in sexuality without entering into an intellectual life that is open to the search for truth and meaning. This openness entails entering into a language that does not settle for scientific-technical rationality to explain human sexuality and also does not base itself on suspicion of any language that engages with teleological explanations of the human being. Only a language with a certain anthropological density can offer educational pathways that allow us to discern which desires are appropriate and which are not, which practices do our nature justice and which degrade it.

2. As sex is an activity between people, it is subject to a specific set of norms, the exigency of which derives from the intimate character that is at stake. Education in sexuality must relate to care for others.

3. If, as we have shown, intimacy is in play when we speak of sexuality, there would be no harm in recovering old terms such as shame, devotion, passion, lust, or concupiscence that enable us, on the one hand, to understand the beauty it contains and on the other the emotions that are mobilised and the risks of a personal rather than health nature that are at stake.

\section{Notes}

${ }^{1}$ It seems likely that the author did not always think the same way in relation to the sexual revolution of our time. In an interesting original text from 1971, Jacinto Choza analyses the suppression of shame in the field of sexuality as a property of our time, and as a manifestation of the suppression of intimacy (Choza, 1990). He was not then conscious of the degree to which this break might have been heightened or caused by the sexual revolution. Nonetheless, the way current adolescents expose themselves in social media and the "transparent" and overexpo. sed world we are creating question the idea that the consequences for intimacy of this revolution are always so positive. It should be noted here that Choza gives shame a definition that is positive and even necessary for understanding intimacy and per. sonal life itself: "Shame is the habit and tendency to maintain possession of one's own intimacy from the most radical instance of the person (the ego), and to maintain this intimacy in the state of the greatest possible perfection, in pursuit of a self-giving through which solitude is transcended and the subject per. fects itself" (Choza, 1990, p. 28).

2 Roger Scruton and Martha Nussbaum had an inter. esting discussion about this point. A summarised version can be found in Reyero (2020).

\section{References}

Barkow, J. H., Cosmides, L., \& Tooby, J. (Eds.) (1992). The adapted mind: Evolutionary psychology and the generation of culture. Oxford University Press.

Bloom, A. (2008). The closing of the American mind. Simon and Schuster.

Brandon, M. (2016). Monogamy and nonmonogamy: Evolutionary considerations and 
treatment challenges. Sexual medicine reviews, 4 (4), 343-352. https://doi.org/10.1016/j. sxmr.2016.05.005

Carter, J., \& Duncan, S. (2017). Wedding paradoxes: Individualized conformity and the "perfect day'. The sociological review, 65 (1), 3-20. https://doi.org/10.1111/1467-954X.12366

Chesterton, G. K. (2005). Questions of divorce. New England Review (1990-), 26 (1), 174-178.

Choza, J. (1990). La supresión del pudor y otros ensayos [The elimination of embarrassment and other essays]. EUNSA.

Choza, J. (2020). El sexo de los ángeles. Sexo y género desde las bacterias a los robots [The sex of the angels. Sex and gender from bacteria to robots]. Editorial Thémata.

Collins, C., Alagiri, P., Summers, T., \& Morin, S. F. (2002). Abstinence only vs. comprehensive sex education: What are the arguments. What is the evidence. AIDS Research Institute, University of California. https://bit.ly/2JAOtHV

Earp, B. D., \& Savulescu, J. (2020). Evolved fragility. In Love is the drug (pp. 101-109). Manchester University Press.

Eberstadt, M. (2019). Primal screams: How the sexual revolution created identity politics. Templeton Foundation Press.

Fine, M., \& McClelland, S. (2006). Sexuality education and desire: Still missing after all these years. Harvard Educational Review, 76 (3), 297-338. https://doi.org/10.17763/ haer.76.3.w5042g23122n6703

Fine, M. (1988). Sexuality, schooling, and adolescent females: The missing discourse of desire. Harvard Educational Review, 58 (1), 29-54. https:// doi.org/10.17763/haer.58.1.u0468k1v2n2n8242

Giroux, H. (2013). Critical pedagogy in dark times. Praxis Educativa, 17 (2), 27-38.

Hadjadj, F. (2010). La profundidad de los sexos. Por una mistica de la carne [The depth of the sexes. For a mystique of the flesh. Nuevo Inicio.

Haidt, J. (2013). Moral psychology for the twenty-first century. Journal of Moral Education, 42 (3), 281-297. https://doi.org/10.1080/030572 40.2013 .817327

Harden, K. P. (2014). A sex-positive framework for research on adolescent sexuality. Perspectives on Psychological Science, 9 (5), 455-469. https://doi.org/10.1177/1745691614535934
Immordino-Yang, M. H., Darling-Hammond, L., \& Krone, C. R. (2019). Nurturing nature: How brain development is inherently social and emotional, and what this means for education. Educational Psychologist, 54 (3), 185-204. https://doi.org/10.1080/00461520.2019.1633924

Irvine, J. M. (2004). Talk about sex: The battles over sex education in the United States. University of California Press.

MacIntyre, A. (1999). Dependent rational animals: Why human beings need the virtues. Open Court Publishing.

Pieper, J. (2011). Guide to Thomas Aquinas. Ignatius Press. https://amzn.to/33B7yks

Reyero D. (2020). Teoría mimética y educación sexual. Contra los géneros en conflict Mimetic theory and sexual education. Against conflictual gender. Xiphias Gladius. Revista Interdisciplinar De Teoría Mimética, 3. https://doi. org/10.32466/eufv-xg.2020.3.635.121-138

Reyero, D., \& Gil Cantero, F. (2019). La educación que limita es la que libera $\mid$ Education that limits is education that frees. revista española de pedagogía, 77 (273), 213-228. https:// doi.org/10.22550/REP77-2-2019-01

Rorty, R. (1989). Education without dogma: Truth, freedom, \& our universities. Dissent, 36, 198-204.

Santelli, J. S., Kantor, L. M., Grilo, S. A., Speizer, I. S., Lindberg, L. D., Heitel, J., Schalet, A. T., Lyon, M. E., Mason-Jones, A. J., McGovern, T., Heck, C. J., Rogers, J., \& Ott, M. A. (2017). Abstinence-only-until-marriage: An updated review of US policies and programs and their impact. Journal of Adolescent Health, 61 (3), 273-280.

Scruton, R. (2006). Sexual desire: A philosophical investigation. Continuum.

Scruton, R. (2009). I drink therefore I am: A philosopher's guide to wine. A\&C Black.

Scruton, R. (2019). On human nature. Princeton University Press.

Symons, D. (1989). A critique of Darwinian anthropology. Ethology and Sociobiology, 10 (1-3), 131-144. https://doi.org/10.1016/0162-3095(89) 90016-2

Spaemann, R. (2003). ¿Es la emancipación un objetivo de la educación? [Is emancipation an aim of education?] In Limites. Acerca de la dimensión ética del actuar (pp.453-465). EIUNSA, 
Spaemann, R. (2017). Prólogo a la edición alemana [Foreword to the German edition]. In G. Kuby (2017), La revolución sexual global. La destrucción de la libertad en nombre de la libertad (pp. 27-29). Didaskalos.

Susman, E. J., Marceau, K., Dockray, S., \& Ram, N. (2019). Interdisciplinary work is essential for research on puberty: Complexity and dynamism in action. Journal of Research on Adolescence, 29 (1), 115-132. https://doi.org/10.1111/jora.12420

Tolman, D. L. (2012). Female adolescents, sexual empowerment and desire: A missing discourse of gender inequity. Sex Roles, 66, 746-757. https://doi.org/10.1007/s11199-012-0122-x

Tolman, D. L., \& McClelland, S. I. (2011). Normative sexuality development in adolescence: A decade in review, 2000-2009. Journal of Research on Adolescence, 21 (1), 242-255. https://doi.or$\mathrm{g} / 10.1111 / \mathrm{j} .1532-7795.2010 .00726 . \mathrm{x}$

Wilson, E. $O$ (2000). Sociobiology: The new synthesis. Harvard University Press

Yela, M. (1996). Comportamiento animal y conducta humana [Animal behaviour and human conduct]. Psicothema, 8, 149-163.

\section{Author biography}

David Reyero García. Doctor of educational sciences from the Universidad Complutense de Madrid and Associate Professor at that university. $\mathrm{He}$ is currently Assistant Head of the Department of Educational Studies and Co-director of the Research Group on Anthropology and Philosophy of Education (GIAFE). He is currently Joint Editor of Revista de Educación published by the Spanish Ministry of Education. His publications have covered aspects of epistemology relating to pedagogical knowledge and current issues relating to new technology, civic education, the politics and economy of education, and its moral aims.

(iD https://orcid.org/0000-0002-9047-532X 